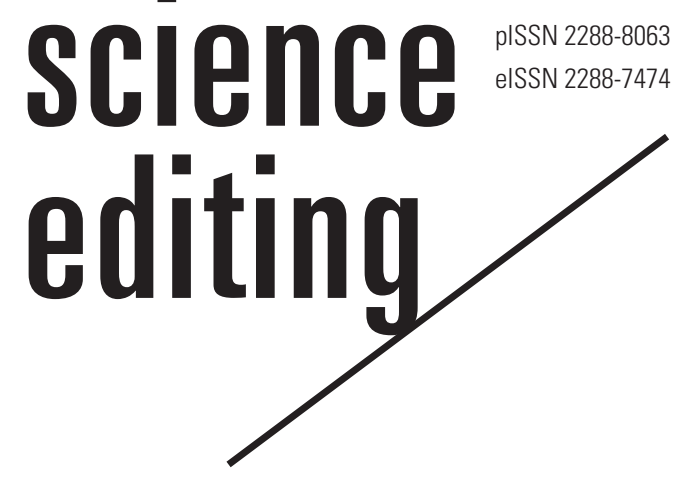

\title{
2018 Asian-Pacific Conference of the International Society of Managing and Technical Editors
}

\author{
Yu-Jin Choi \\ Department of Neuropsychiatry, Kyung Hee University College of Korean Medicine, Seoul, Korea
}

I attended the Asian-Pacific Conference of the International Society of Managing and Technical Editors in Singapore on March 27 to 28, 2018. I had the opportunity to attend this conference, which provided an excellent venue for learning and exchanging ideas regarding current issues and technologies related to journal publishing, after passing the examination for the Korea Manuscript Editors Certification and completing the basic training course for manuscript editors organized by the Korean Council of Science Editors in 2017. The most notable topics discussed at the conference included the launch of a new platform, a preprint server, which is different from the traditional form of journal publishing; the development of new technologies in the journal review process to complement the traditional peer review system; and ways to promote research papers and journals to enhance their impact (Fig. 1).

A preprint is a scholarly article that is uploaded online before it is peer-reviewed and published in an academic journal. Preprint servers, distinct from academic journals, distribute preprint research papers. Previously, this system has been used in the fields of physics and mathematics, and interest has emerged in applying it in the medical field. However, some concerns have also been raised regarding the preprint system. At the conference, 3 speakers presented on their experiences and points to consider regarding preprint servers in their fields of study (biology, medicine, and chemistry), and a discussion ensued. Lastly, a discussion on the ethical issues regarding preprints raised by the Committee on Publication Ethics followed. The presentations were interesting, and it was productive to learn about various perspectives from each field of study.

In the fields of physics, mathematics, and computer science, the preprint server arXiv.org, was introduced in 1991 and has been active since then. In the life sciences, bioRxiv.org was

Received: June 4, 2018 Accepted: July 13, 2018

Correspondence to Yu-Jin Choi choiyujin@khu.ac.kr

ORCID

Yu-Jin Choi

http://orcid.org/0000-0001-5257-2340 launched in November 2013. Some journals, including those published by the American Society for Microbiology and the British Medical Journal, developed a bioRxiv journal submission system, in which authors post a research paper on bioRxiv first, and then submit it to a journal. Based on the bioRxiv model, medRxiv will be developed in the second quarter of 2018. John Inglis, the co-founder of bioRxiv and medRxiv, was the first speaker and discussed the launch of bioRxiv and plans for medRxiv.

In general, it takes a long time to publish a peer-reviewed paper. Therefore, the most promi- 

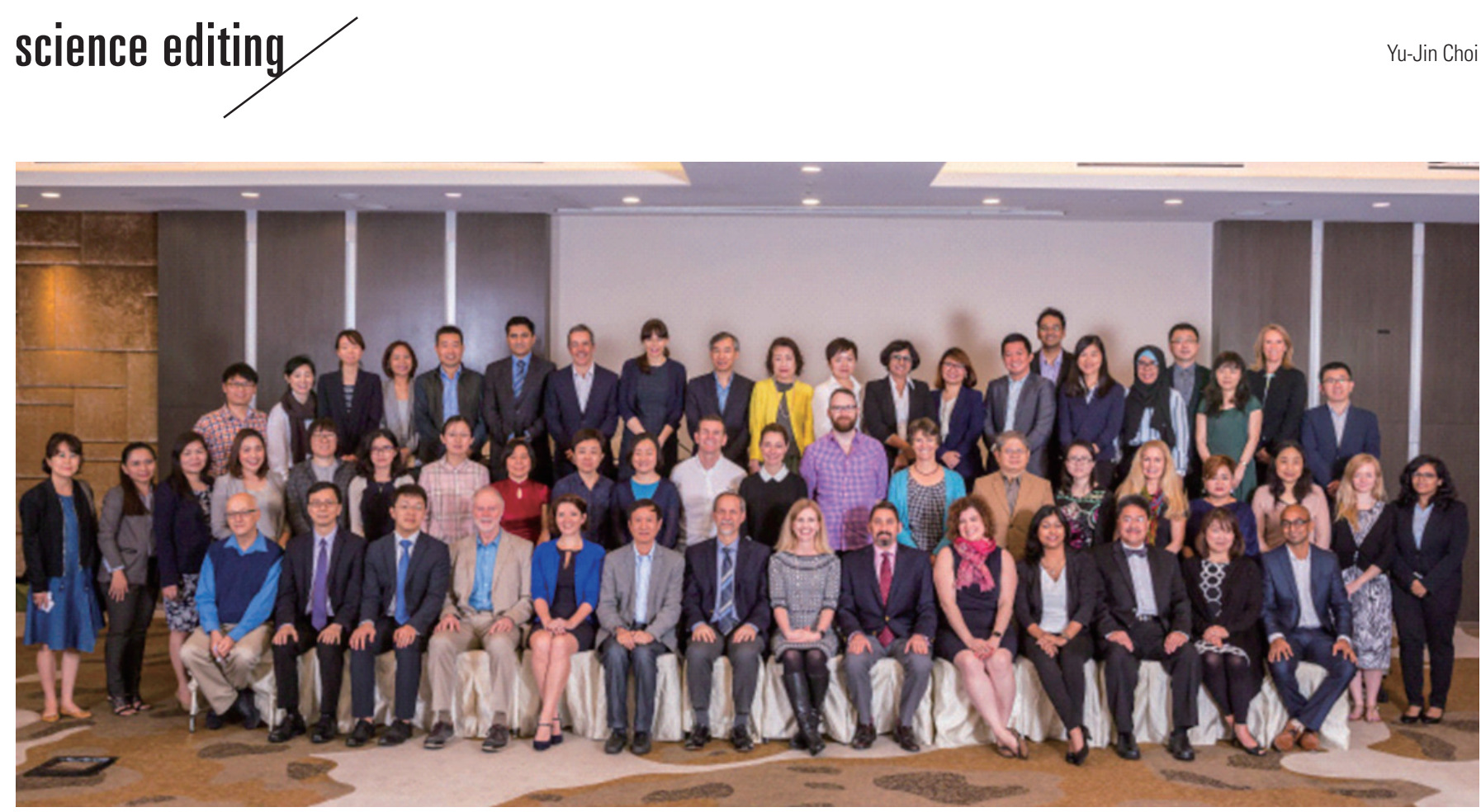

Fig. 1. Group photo from the 2018 Asian-Pacific Conference of the International Society of Managing and Technical Editors.

nent benefit of the preprint system is that it enables authors to share their latest research findings immediately with the scientific community. Furthermore, authors can post and share their research findings for free, or only at a minimal cost, and receive feedback from many readers. However, there are many concerns about adopting the preprint system in the medical field. Since preprints are published without a formal peer review process, one main concern is that unverified information could be widely spread.

In order to minimize the risk of publishing articles without a peer review process, medRxiv is planning to strictly screen articles in accordance with standard criteria. In addition, a number of other related questions were brought up, including (1) whether preprints can be accepted as published research, (2) how to solve problems of copyright and citations of a preprint article once a research paper has been published in an academic journal, and (3) whether academic journals would accept preprint articles, which was a concern from the authors' point of view. In the presentation, it was suggested that these concerns could be addressed at least in part by clearly indicating that preprints have not been peer-reviewed and by ensuring that preprint servers implement strict screening standards for preprints. Further discussion on preprints can be found on the Committee on Publication Ethics website.

I was surprised to learn that artificial intelligence plays a significant role in journal publishing. In fact, most journals have already been applying artificial intelligence to check for plagiarism. Since a human being has limitations in detecting plagiarism through memory, a system with an immense data- base has been checking for plagiarism by comparing submitted texts with existing publications. StatReviewer is a novel system that was developed by a statistician to check whether appropriate statistical methods have been properly applied in a research paper. It was also reported that StatReviewer can check whether mandatory items that should be reported in clinical trials have all been properly reported.

The significance of an article should still be evaluated by a human being, but using a computer system in parallel would improve the effectiveness of evaluating articles for plagiarism, whether various items were reported in accordance with guidelines, and whether appropriate statistical methods were used. Of course, a qualified human being would still need to check the findings generated by the system. StatReviewer has not yet been widely implemented. However, the beta version was said to be available, which made me want to use this system to check my own research papers. The Q\&A session was flooded with enthusiastic responses from the audience. Just as plagiarism checking programs are widely implemented by most journals, StatReviewer may also be widely applied to check submitted manuscripts in the future.

As more and more articles and journals are inundating the world of research, one of the most important topics at the conference was how to better promote research papers or journals. A presentation about successful journal promotion using WeChat in China was particularly impressive. WeChat is one of the most popular social networking services in China; for example, the Chinese Journal of Natural Medicine has an official account on WeChat, and it promotes its latest arti- 
cles and introduces journal editors via WeChat. The presenters reported that using this platform increased the exposure frequency and rank of the journal. Additionally, many journals are using Twitter to promote themselves.

Recently, many journals have started to provide figures that capture the content of their articles or video articles to communicate their findings more effectively and to make them stand out from other articles. A presentation on the experience of using data visualizations by the Korean journal, Annals of Laboratory Medicine was impressive. This journal provides video summaries to improve the readability of articles and uploads them on YouTube to increase exposure and to help readers easily grasp the contents of articles. From the readers' point of view, graphical abstracts are helpful to understand the content at a glance.

Kudos (http://growkudos.com/) is a website that has been developed to effectively disseminate articles to the general public. The website provides accessible titles and content summaries, along with links to the original articles.

Research advances continuously. However, I initially thought that the publication process would not change dramatically in response to new technological developments, since the journal publication process - in which authors write papers about their new research findings, experts in the field review and select articles for publication, and the manuscripts are published in an academic journal-is simple and clear. By attending this conference, however, I learned that the journal publication process is constantly changing and stands at the forefront of the development of new technologies and improvements.

\section{Conflict of Interest}

No potential conflict of interest relevant to this article was reported.

\section{Acknowledgments}

This work was supported by a travel grant from the Korean Council of Science Editors (2018). 